\title{
Animal models of medullary thyroid cancer: state of the art and view to the future
}

\author{
Giovanni Vitale1,2, Germano Gaudenzi', Luisa Circelli3, Marco F Manzoni4, \\ Andrea Bassi ${ }^{5}$, Niccolò Fioritti6, Antongiulio Faggiano7, Annamaria Colao8 \\ and on behalf of NIKE Group
}

1Department of Clinical Sciences and Community Health (DISCCO), University of Milan, Milan, Italy 2Laboratory of Endocrine and Metabolic Research, Istituto Auxologico Italiano IRCCS, Milan, Italy ${ }^{3}$ Department of Experimental Oncology, Laboratory of Molecular Biology and Viral Oncology, Istituto Nazionale per lo Studio e la Cura dei Tumori, 'Fondazione Pascale' - IRCCS, Naples, Italy ${ }^{4}$ Department of Endocrinology and Internal Medicine, Endocrine Tumors Unit, San Raffaele Hospital Vita-Salute San Raffaele University, Milan, Italy ${ }^{5}$ Department of Physics, Politecnico di Milano, Milan, Italy ${ }^{6}$ Department of Biosciences, University of Milan, Milan, Italy 7Thyroid and Parathyroid Surgery Unit, Istituto Nazionale per lo Studio e la Cura dei Tumori 'Fondazione G. Pascale' - IRCCS, Naples, Italy ${ }^{8}$ Department of Clinical Medicine and Surgery, Section of Endocrinology, 'Federico II' University of Naples, Naples, Italy

Correspondence should be addressed to $\mathrm{G}$ Vitale Email giovanni.vitale@unimi.it

\begin{abstract}
Medullary thyroid carcinoma is a neuroendocrine tumour originating from parafollicular $C$ cells accounting for $5-10 \%$ of thyroid cancers. Increased understanding of diseasespecific molecular targets of therapy has led to the regulatory approval of two drugs (vandetanib and cabozantinib) for the treatment of medullary thyroid carcinoma. These drugs increase progression-free survival; however, they are often poorly tolerated and most treatment responses are transient. Animal models are indispensable tools for investigating the pathogenesis, mechanisms for tumour invasion and metastasis and new therapeutic approaches for cancer. Unfortunately, only few models are available for medullary thyroid carcinoma. This review provides an overview of the state of the art of animal models in medullary thyroid carcinoma and highlights future developments in this field, with the aim of addressing salient features and clinical relevance.
\end{abstract}

\section{Key Words}

- medullary thyroid cancer

- animal models

- mice

- zebrafish

- Drosophila melanogaster

- chick chorioallantoic membrane

Endocrine-Related Cancer (2017) 24, R1-R12

\section{Introduction}

Medullary thyroid carcinoma (MTC) is a neuroendocrine tumour originating from parafollicular $\mathrm{C}$ cells. At the end of 60s, after the discovery that MTC represents a unique thyroid cancer, it was recognised that the tumour occurred either sporadically or in a hereditary form as a component of the type 2 multiple endocrine neoplasia (MEN) syndromes, MEN2A and MEN2B, and the related syndrome, familial MTC (FMTC). RET (REarranged during Transfection) proto-oncogene alterations are crucial events for MTC development. The most frequent RET alterations in MTC are activating point mutations, but deletions and insertions have also been reported (Romei et al. 2016). The clinical course of patients with MTC is variable, ranging from indolent to extremely aggressive, 
and it is related to the stage of the disease at the time of diagnosis (Vitale et al. 2001). Ten-year survival rates for patients with stages I, II, III and IV MTCs are 100\%, 93\%, 71\% and 21\%, respectively (Modigliani et al. 1998). In the last decades, there has been no significant trend towards an earlier stage of disease at the time of diagnosis. Indeed, under half of the patients present with stage III or IV disease (Kebebew et al. 2005, Roman et al. 2006). Surgery is the only curative treatment for MTC. Radiotherapy and chemotherapy play a marginal role in advanced MTC (Vitale et al. 2001, Cabanillas et al. 2016). Somatostatin analogues are able to control the neuroendocrine symptoms induced by advanced MTC (Lupoli et al. 1996, Vitale et al. 2000). In recent years, several tyrosine kinase inhibitors (axitinib, cabozantinib, gefitinib, imatinib, motesanib, sorafenib, sunitinib and vandetanib) have been evaluated in phase I, II and III clinical trials of patients with advanced MTC. These drugs increased progression-free survival, however, with time, the MTC becomes resistant to these drugs and the disease progresses (Cabanillas et al. 2016, Viola et al. 2016). In addition, few preliminary clinical studies evaluated the potential beneficial effects of mTOR inhibitors in the treatment of MTC, showing the stabilisation of disease in most of patients treated with everolimus (Druce et al. 2012, Faggiano et al. 2012, Lim et al. 2013, Schneider et al . 2015). Therefore, in patients with advanced MTC, there is a critical need for more effective therapies.

Animal models are indispensable tools for investigating the pathogenesis, mechanisms for tumour invasion and metastasis and new therapeutic approaches for cancer. Unfortunately, only few models are available for MTC. This paper provides a state-of-the-art overview of animal models in MTC and highlights the future developments in this field, with the aim of addressing the salient features and clinical relevance.

\section{Murine models}

In the last year, several genetically engineered murine models of MTCs have been constructed to replicate this disease and related variants. These models were generated by overexpressing mutated oncogenic RET or other oncogenes in transgenic mice or through the loss of tumour suppressor genes (Cote et al. 2015, Kirschner et al. 2016, Wiedemann \& Pellegata 2016).

Another traditional preclinical model consists of implantation of MTC cell lines or tumour fragments into immunodeficient rodents.

\section{Genetically engineered mouse models carrying RET mutations}

In humans, activating mutations of RET proto-oncogene play a relevant role in the pathogenesis of both familial (MEN2A, MEN2B and FMTC) and sporadic forms of MTC. Germline activating mutations of RET have been reported in around $88-98 \%$ of familial MTCs, whereas somatic mutations of this gene have been detected in $23-70 \%$ of sporadic forms (Manfredi et al. 2016). The RET protooncogene encodes a tyrosine kinase transmembrane receptor, which is mainly expressed in neural crest-derived cell lineages, such as parafollicular C cells, and is involved in the control of cell differentiation and proliferation. The RET COOH-terminal tail varies in length as a result of alternative splicing of the $3^{\prime}$ end, generating three different isoforms: RET9, RET43 and RET51. Although isoforms share the majority of signalling potentials, there are differences in phosphorylation and binding of signalling molecules (Myers et al. 1995, Santoro \& Carlomagno 2013).

Several murine models have been developed to study the pathogenesis and progression of MTC through the use of genetic engineering techniques targeting the Ret gene (Table 1).

The most available mouse models of MTC were generated by tissue-specific expression (mainly in thyroid C cells) of mutated Ret gene. Michiels and coworkers generated transgenic mouse lines expressing the RET9 isoform with the MEN2A-associated point mutation at codon 634 (Michiels et al. 1997). In these animals, the expression of the mutated RET gene was targeted to the thyroid $\mathrm{C}$ cells under the control of the calcitonin generelated peptide (CGRP)/calcitonin (CT) rat promoter. Mice expressing the transgene in the thyroid $\mathrm{C}$ cells displayed bilateral C-cell hyperplasia followed by the development of multifocal and bilateral MTC with a complete penetrance at 14 months in two transgenic mouse lines, and with an incomplete penetrance in one transgenic line. These tumours were morphologically and biologically similar to human MTC, demonstrating that the short isoform of RET is able to drive tumorigenesis in vivo.

Reynolds and coworkers reported that mice carrying RET51 isoform with the C634R mutation under the control of the human CT promoter developed not only MTC but also follicular tumours similar to human papillary thyroid carcinoma (Reynolds et al. 2001). These mice developed C-cell hyperplasia by 3 months of age (35\% of cases), and then bilateral MTC by 6-12 months, with a frequency of $52 \%$, which raised to $68 \%$ in mice over 12 months of age.

Published by Bioscientifica Ltd 
Table 1 Genetically engineered mouse models of medullary thyroid cancer carrying RET mutations.

\begin{tabular}{|c|c|c|c|c|c|}
\hline RET mutation & RET isoform & Promoter & Background strain & Tumor phenotype & Reference \\
\hline C634R & RET9 & Rat CT & C57BL/6J_DBA2 & $\begin{array}{l}\mathrm{CCH} ; \text { multifocal and bilateral } \\
\text { MTC }\end{array}$ & Michiels et al. (1997) \\
\hline C634R & RET51 & Human CT & CBA/B6/FVB & $\begin{array}{l}\text { CCH, bilateral MTC; human } \\
\text { PTC; exocrine pancreatic } \\
\text { tumours }\end{array}$ & Reynolds et al. (2001) \\
\hline C634R & RET51 & Human CT & $\mathrm{BALB} / \mathrm{C}$ & MTC (14\%) & Cranston \& Ponder (2003) \\
\hline C634R & RET51 & Human CT & C57BL/6J & MTC (64\%) & Cranston \& Ponder (2003) \\
\hline C634R & RET51 & Human CT & $\mathrm{CBA} / \mathrm{Ca}$ & MTC (98\%) & Cranston \& Ponder (2003) \\
\hline M918T & RET9 & Human CT & C57BL/6J_DBA2 & $\begin{array}{l}\mathrm{CCH} \text {, multifocal and bilateral } \\
\text { MTC }\end{array}$ & Acton et al. (2000) \\
\hline M919T* (Heterozygous) & - & - & C57BL/6J & $\begin{array}{l}\mathrm{CCH} \text {; adrenal chromaffin cell } \\
\text { hyperplasia; } \mathrm{PHE}\end{array}$ & Smith-Hicks et al. (2000) \\
\hline M919T* (Homozygous) & - & - & C57BL/6J & $\begin{array}{l}\mathrm{CCH} ; \mathrm{PHE} \text {; ganglioneuromas of } \\
\text { the adrenal medulla }\end{array}$ & Smith-Hicks et al. (2000) \\
\hline
\end{tabular}

$\mathrm{CCH}, \mathrm{C}$-cell hyperplasia; CT, calcitonin; MTC, medullary thyroid carcinoma; PHE, pheochromocytoma; PTC, papillary thyroid carcinoma.

*Mutation in murine RET gene.

This age-dependent tumour progression resembles the situation in MEN2 patients.

In a study using the CGRP-RET C634R model of MTC, a strong variation was observed in tumour penetrance between mouse background strains, with tumours in $0 \%$ of $\mathrm{FVB} / \mathrm{N}$ mice, $14 \%$ of $\mathrm{BALB} / \mathrm{c}$ mice, $64 \%$ of $\mathrm{C} 57 \mathrm{BL} / 6 \mathrm{~J}$ mice and $98 \%$ of CBA/Ca mice all backcrossed from a single transgenic founder (Cranston \& Ponder 2003). Tumour size positively correlated with the degree of penetrance. This study highlights the role of modifier genes in disease phenotype and the need for careful consideration during selection of mouse background strains (Cranston \& Ponder 2003).

Acton and coworkers created tissue-specific transgenic mice expressing the human RET oncogene cDNA encoding the RET9 isoform in which the MEN2B-specific M918T mutation was introduced (Acton et al. 2000). In humans, MEN2B was almost exclusively associated with the M918T alteration in exon 16 of RET gene. In addition, M918T is the most frequent RET somatic point mutation observed in sporadic MTC (Romei et al. 2016). Transgenic mice carrying human RET mutation developed bilateral MTC in three out of eight founder lines at 20-22 months of age and showed high CT levels (Acton et al. 2000).

Another mouse model for the MEN2B syndrome variant was created by Smith-Hicks and coworkers by introducing into the mouse Ret gene the M919T codon alteration, which corresponds to the M918T mutation in humans (Smith-Hicks et al. 2000). The heterozygous transgenic mice (Ret $\left.{ }^{\mathrm{MEN} 2 \mathrm{~B} /+}\right)$ developed thyroid C-cell hyperplasia (in $31 \%$ of mice at the age of $4-7$ months, in $55 \%$ at $8-12$ months); adrenal chromaffin cell hyperplasia (in $17 \%$ of mice at the age of $4-7$ months, in $16 \%$ at $8-12$ months); and pheochromocytoma (in $2 \%$ at the age of 8-12 months). Homozygous mutant mice (Ret ${ }^{\mathrm{MEN} 2 \mathrm{~B} / \mathrm{MEN} 2 \mathrm{~B})}$ displayed more severe thyroid lesions than to $\operatorname{Ret}^{\mathrm{MEN} 2 \mathrm{~B} /+}$ mice: C-cell hyperplasia (in $86 \%$ of mice at the age of 6-10 months), which however did not progress to MTC; and pheochromocytomas (in 100\% of mice at the age of 6-10 months). Surprisingly, neither multiple mucosal neuromas nor ganglioneuromas of the gastrointestinal tract, tumours typically used to distinguish between MEN2A and MEN2B, could be found in either heterozygous or homozygous mutant mice. However, ganglioneuromas of the adrenal medulla and enlargement of the associated sympathetic ganglia have been observed in 100\% of homozygous mutant mice. These data suggest that the homozygous mice could represent the human MEN2B syndrome more closely than the heterozygous ones, showing an early incidence and increased severity of C-cell hyperplasia and chromaffin cell hyperplasia. However, the homozygous mice are different from the human MEN2B for two main aspects: they did not develop MTC (up to 12 months of age) or ganglioneuromas of the gastrointestinal tract.

Altogether, these different genetically engineered mouse models provided compelling evidence that specific mutations of RET proto-oncogene are oncogenic in parafollicular $\mathrm{C}$ cells. Moreover, they offer the possibility to study the early stages of tumour formation (i.e. C-cell hyperplasia), which is very difficult to perform in patients and could be useful to test novel drugs capable of inhibiting RET function in immunocompetent animals. However, there are also few limitations in using genetically engineered mouse models, mainly related to

Published by Bioscientifica Ltd. 
species-specific differences and to the complexity of the human tumour that cannot be reliably mimicked.

\section{CGRP-v-Ha-ras mouse model}

RAS is a family of related proteins consisting of small GTPases that act as binary molecular switches that control intracellular signalling network. They are mainly activated through growth factors binding to tyrosine kinase receptors, such as RET. Point mutations in RAS (mainly H-RAS and K-RAS) have been reported in sporadic $R E T$-negative MTC with a percentage varying from $0 \%$ to $43.3 \%$, suggesting that activation of these proto-oncogenes represents alternative genetic events in sporadic MTC tumorigenesis (Moura et al. 2015).

Transgenic mice of a C57BL/6 3 SJL strain expressing $v$-Ha-ras in C cells under control of the CGRP promoter developed C-cell hyperplasia progressing to MTC in $85 \%-93 \%$ of animals (depending on founder line) between the ages of 6 and 12 months (Johnston et al. 1998). Tumours were able to secrete CT. Metastasis occurred rarely to the lung and cervical lymph nodes. Some tumours had amyloid deposition, cystic cavitation and vascular invasion and haemorrhage, characteristic features of human MTC. In 4 out of 22 tumours, immunohistochemical staining was positive both for CT and thyroglobulin. Therefore, this transgenic mouse represents an interesting model for investigating the role of RAS in the pathogenesis of MTC and also for elucidating the mechanisms related to the development of mixed medullary-follicular thyroid carcinoma.

\section{Mouse models with mutations in cell-cycle control genes}

A significant number of sporadic MTCs resulted to be negative for RET or RAS mutations. Therefore, other genes also appear to be involved in the pathogenesis of this tumour (Romei et al. 2016).

The retinoblastoma $(R B)$ and p53 genes encode tumour suppressors that are crucial in the regulation of cell cycle. Loss or mutations of these genes have been reported in many types of human cancers. It was found that mice bearing a single $R b$ mutant allele $\left(R b^{+-}\right)$develop pituitary tumours with almost complete penetrance (Cote et al. 2015, Kirschner et al. 2016). $R b^{+/-}$mice were also predisposed to develop C-cell hyperplasia, which subsequently progressed into MTC. Depending on the specific recombinant mouse mutant used and the genetic background, the incidence of these tumours ranged from 50\% to 90\% (Williams et al. 1994, Harrison et al. 1995,
Park et al. 1999, Ziebold et al. 2003). These tumours were mostly small, predominantly unilateral and nonaggressive.

Interestingly, Ziebold and coworkers reported that 56\% of $R b^{+/-}$mice with normal $p 53$ status on a 129/Sv 3 C57BL/6 background developed C-cell hyperplasia or MTC, and rare metastases to liver, lung, lymph node and adrenal gland (Ziebold et al. 2003). The growth-suppressive properties of $\mathrm{RB}$ are largely dependent on its ability to regulate the E2F transcription factors. The concomitant inactivation of the E2F3 transcription factor increases the aggressiveness of the MTC yielding metastases at a high frequency.

Another study showed that about $37 \%$ of mice (a mixed inbred C57BL/6 and 129/Sv genetic background) heterozygous for both $R b$ and $p 53$ mutant alleles $\left(R b^{+/-}\right.$ $p 53^{+-}$) developed C-cell hyperplasia and MTC (Harvey et al. 1995). $R b^{+/-}$and $p 53^{+/-}$mice also developed several non-thyroidal neoplasms and had shortened life spans. Spontaneous activating mutations of Ret were detected in 4 out of 8 MTCs from $\mathrm{Rb}^{+/-} \mathrm{p5}^{+/-}$mice (Coxon et al. 1998). These findings demonstrate the ability of the gene knockout model to recapitulate somatic multi-step tumorigenesis in MTC.

Recently, a new genetically engineered mouse model was generated to determine the role of $R b$ and p53 in MTC carcinogenesis and progression (Akeno et al. 2015). Mice were developed to target Cre recombinasemediated $R b$ gene ablation under the control of the surfactant protein C (SPC) promoted reverse tetracycline transactivator. Treatment with doxycycline throughout gestation resulted in $R b$ ablation throughout the lung epithelium as well as in a subset of thymic and thyroid cells. Double transgenic mice homozygous for floxed $R b$ alleles (designated $R b$ ablated) developed pulmonary neuroendocrine hyperplasia and thyroid tumours expressing CT. Moreover, p53 gene was conditionally ablated in $R b$-deficient thyroid by generating double transgenic mice with floxed $R b$ and $p 53$ alleles. The additional $p 53$ loss resulted in dramatic MTC progression. Overall, this study demonstrated that $R b$ loss is sufficient to induce thyroid tumours that phenotypically mimic human MTC and that although p53 loss is not sufficient to initiate neuroendocrine tumourigenesis, p53 plays a critical role in suppressing $R b$-deficient cancer progression.

$\mathrm{RB}$ exerts its tumour suppressor activity through interactions with the cyclin-dependent kinases (CDKs), which are also inhibited by a family known as the CDK inhibitors (CDKNs). Several studies evidenced the spontaneous development of C-cell hyperplasia in mice lacking CDKN2C (Franklin et al. 2000, van Veelen et al. 2008) or CDKN1B (Franklin et al. 2000).

Published by Bioscientifica Ltd 
Pozo and coworkers recently generated a mouse model, in which MTC proliferation was induced upon conditional overexpression of the CDK5 activator, $p 25$, in $\mathrm{C}$ cells (under the control of the neuron-specific enolase promoter) and arrested by interrupting p25 overexpression (Pozo et al. 2013). Analysis of the mechanism demonstrated that p25 overexpression led to enhanced CDK5 kinase activity, leading to the phosphorylation and inactivation of RB.

However, these models may not faithfully mimic the human situation. Indeed, data concerning RB and p53 loss in human MTC are scanty and contradictory; therefore, their role in the development of spontaneous MTC is still unclear (Cerrato et al. 2009). Holm and Nesland (1994) reported in all MTCs (46 cases) a heterogeneous RB protein nuclear staining, suggesting that inactivation of the RB gene is not important for the pathogenesis of MTC. Although in another series, three of the six cases of MTC (50\%) showed loss of expression of RB protein (Anwar et al. 2000). Using the COSMIC database, CDKN2C somatic mutations have been reported in $8 \%$ of MTC samples (http://cancer.sanger.ac.uk/cosmic). In addition, a recent paper showed that the detection of somatic CDKN2C loss was associated with the presence of distant metastasis and decreased the overall survival in a cohort of 62 patients with sporadic MTC (Grubbs et al. 2016). Eight out of 15 (53\%) MTC harboured single point mutation or loss of heterozygosity in p53 locus (Pavelić et al. 2006). In another series of MTC, allelic loss of p53 has been reported in $44 \%$ of cases (Sheikh et al. 2004).

\section{MTC xenograft murine model}

Xenograft tumour models have been widely used as an in vivo counterpart to cell lines by cancer researchers. In the last years, MTC xenograft mouse model has been adopted as preclinical human thyroid cancer model to test the efficacy and the safety of several kinase inhibitors.

In most cases, to test the in vivo activity of several drugs or drug combination, TT or MZ-CRC-1 cells (both human MTC cell lines bearing mutations in protooncogene RET) were inoculated subcutaneously (sc) on the back of athymic nu/nu mice. When tumours became palpable and mean tumour volume was approximately $70-80 \mathrm{~mm}^{3}$, animals were randomly divided into control group and treated group (Broutin et al. 2011, Bentzien et al. 2013, Lopergolo et al. 2014, Tohyama et al. 2014).

Indeed, a strong antitumor effect of sunitinib and other multi-kinase inhibitors, such as vandetanib or sorafenib, has been observed in vivo in TT cells implanted in mice (Broutin et al. 2011). The antitumor efficacy of sunitinib was also tested in mice carrying MZ-CRC-1 xenografts cotreated with cisplatin, showing that the combination of the two drugs was able to effectively reduce the tumour mass, resulting in enhanced antitumor activity (Lopergolo et al. 2014). In a recent study, the in vivo pharmacodynamic experiments showed substantial inhibition of RET in TT xenograft tumours after a single oral dose of cabozantinib, a potent RET inhibitor. In nude mice, cabozantinib was well tolerated and resulted in a dose-dependent tumour growth inhibition correlated with a reduction in circulating CT levels (Bentzien et al. 2013). To date, cabozantinib and vandetanib have been approved by FDA for the treatment of patients with progressive, metastatic or unresectable MTC. The antitumor efficacy of lenvatinib, a VEGF inhibitor, was also tested in TT xenograft model. This molecule showed to inhibit the in vivo tumour growth of TT xenografts in a dose-dependent manner. Together with the antitumor activity, lenvatinib showed a potent inhibition in tumour-induced angiogenesis (Tohyama et al. 2014).

These findings provide evidence of xenograft model as a potential preclinical model to investigate the antitumor activity of new compounds in MTC. However, it should be taken into consideration that the transplantation of immortalised tumour cell lines provides a simplified cancer model with limited applications in the clinical setting (Rubio-Viqueira \& Hidalgo 2009).

Another type of xenograft model used in research is the 'patient-derived tumour xenograft' (PDX). This model is based on the transfer of primary tumour directly from the patient into an immunodeficient mouse. To accomplish this, patient tumours must be obtained fresh from surgery, at which point they are mechanically or chemically digested, with a small portion saved as a primary stock, and established in a NOD-SCID mouse. PDX models are maintained by passaging cells directly from mouse to mouse once the tumour burden becomes too high. Tumours can be engrafted heterotopically or orthotopically. Heterotopic PDX models involve implanting tumours into the subcutaneous flank of a mouse. This method allows for easier cell transfer and precise monitoring of tumour growth and location (Kim et al. 2009). Orthotopic models are more technically challenging and time consuming. This method involves the direct implantation to the mouse organ of choice. In some cases, additional imaging studies may be needed to verify the location of tumour grafts after implantation. Orthotopic transplants are considered to more accurately

Published by Bioscientifica Ltd. 
mimic the human tumours from which they are derived than heterotopic transplants when comparing histology and gene expression profiles from mice to patients. PDX models appear to be superior to the implantation of cancer cell lines because they maintain more similarities to the parental tumours. In addition, PDX represents a more physiologically relevant model compared to $2 \mathrm{D}$ in vitro cellular models, where primary cultures of cancer cells grow in suspension culture, or as monolayers on plastic surfaces. Indeed, PDX murine models mimic more accurately the tumour microenvironment and the complex biochemical and physical interactions between the cancer cells and the stromal components (RubioViqueira \& Hidalgo 2009). However, for MTC, there are serious difficulties to generate a murine PDX model due to the slow tumour growth of MTC in most of the cases and the low engraftment rate of this tumour in mice, representing an important shortcoming for this model. This could be the reason why in literature, we found only one paper reporting the use of PDX murine model in MTC (Gyory et al. 2005).

\section{Drosophila cancer model}

In addition to the existing murine models that remain the gold standard, other animal organisms have been used in the preclinical research on MTC. Even if the number of these alternative models is still limited, their applications appear suitable and promising.

The fruit fly Drosophila melanogaster has contributed significantly in several fields of biology as the genetic organism of choice for studying epithelial development. In the last years, Drosophila has proven useful in establishing genetic models of human diseases due to its intrinsic features as the low cost of maintenance, the reduced genetic redundancy, the high conservation of human signalling pathways and disease genes and the ease of mutant flies generation. The fact that Drosophila contains functional homologs for the $68 \%$ of human cancer-related genes, including the proto-oncogene RET, has provided a rationale for using this model in tumour pathogenesis studies and in anti-cancer drug discovery (Yadav et al. 2016). In this frame, Drosophila has been used to study MEN2 syndrome by the generation of transgenic flies that expressed mutated forms of Drosophila RET (dRet) in the developing retina, a neuroepithelial tissue that shows remarkable evolutionary conservation of receptor tyrosine kinase signal transduction pathways. Interestingly, the expression of MEN2A and MEN2B analogue mutations led to aberrant cell proliferation and inappropriate cell fate specification in developing Drosophila retina. Moreover, it has been demonstrated that components of the Ras/ERK, Src and JNK pathways, previously linked to the vertebrate MEN2 signalling, are required for the phenotypic outcomes of $\operatorname{dRet}^{\mathrm{MEN} 2}$ activity, thereby validating the Drosophila models (Read et al. 2005). Some experiments have taken advantage of these transgenic models to perform a screen for genes that dominantly suppress or enhance dRet ${ }^{\mathrm{MEN} 2}$ phenotypes. In this context, $\sin 3 a$, which negatively regulates the transcription of a wide array of genes (van Oevelen et al. 2008), emerged as a new important mediator of dRet ${ }^{\mathrm{MEN} 2}$ (Read et al. 2005). The dRet transgenic models have also been used to quickly and effectively identify compounds of potential therapeutic value for MTC. When dRetMEN2B larvae were fed vandetanib (Vidal et al. 2005) or sorafenib (Das \& Cagan 2010), both multi-kinase inhibitors targeting Ret induced a suppression of the mutant phenotype. In addition, Drosophila models of RET fusions (CCDC6-RET and NCOA4-RET) have been recently generated to study the specific pathway activated by RET fusion isoforms and to test their sensitivity to clinically relevant drugs (Levinson \& Cagan 2016). This elegant strategy represents a new tool to identify the patterns of drug sensitivity for each RET isoform.

In conclusion, Drosophila offers powerful tools to investigate the molecular basis of MTC and to perform high-throughput screening of novel cancer drugs, as well as to test multi-targeted agents and combination therapy (Dar et al. 2012, Das et al. 2014, Yadav et al. 2016). However, the employment of Drosophila for studies on MTC has several limitations that need to be considered. Indeed, the short life cycle can limit the study of sequential step-wise changes that usually are specific of slow growing tumours, such as MTC. Nonetheless, the study of tumour progression, which may rely on tissuespecific conditions, may be compromised by the lack of thyroid and C cells-like structures (Das \& Cagan 2010). Moreover, given that the anatomy and physiology of flies are significantly different from those of mammals, it will be important to clarify some issues related to the potential efficacy in humans of drugs that have been tested in Drosophila (Yadav et al. 2016).

\section{Chick chorioallantoic membrane (CAM)}

Among the in vivo models, the chick embryo chorioallantoic membrane (CAM) has been used to implant several 
tumours to study cell proliferation, angiogenic and metastatic behaviour and to test the antitumor activity of novel compounds (Ribatti 2014).

Yalcin and coworkers adopted this model for MTC. Human MTC cells, grafted in the proximity of the chick chorioallantoic membrane (CAM), were able to quickly stimulate the neovascularisation. In particular, this model was used as a parallel platform to xenografts in nude mice to confirm the antiangiogenic and antiproliferative effects of tetraiodothyroacetic acid on human MTC implanted cells (Yalcin et al. 2010). Although the CAM test is a relatively simple, quick and cost-saving angiogenesis assay, there are also important limitations that must be taken into account: (1) a large number of eggs are required to obtain consistent results; (2) it could be difficult to distinguish tumour-induced vessels from a falsely increased vascular density due to rearrangement of existing vessels; (3) despite the immaturity of the chick immune system, a nonspecific inflammatory reaction can occur particularly if experiments are extended after 15 days of incubation (Staton et al. 2009, Ribatti 2016).

\section{Zebrafish}

Another powerful animal model that can help the preclinical research on MTC is zebrafish (Danio rerio). This small teleostean fish emerged as a model organism for developmental biology studies due to its intrinsic features as the high fecundity, the outer fertilisation and the conservation of morphogenetic pathways that regulate the development of organs from fish to mammals (White et al. 2013). The optical transparency of embryos and larvae, together with the availability of transgenic lines that specifically express fluorescent proteins in different tissue and organs, makes zebrafish a particularly suitable model for in vivo imaging of developmental events through different microscopy techniques. A growing number of publications suggest that zebrafish is a unique in vivo system to faithfully recapitulate human cancer through carcinogenic induction, transgenesis, gene inactivation and xenotransplantation (Huiting et al. 2015).

In this frame, zebrafish could be used to investigate the molecular aspects of both pathogenesis and tumour progression in MTC. Indeed, zebrafish ret gene encode for two isoforms, Ret9 and Ret51, that share high amino acid identity with the human counterparts (Marcos-Gutierrez et al. 1997, Lucini et al. 2011), in particular, at the level of tyrosine kinase domain (Fisher et al. 2006). Recently, the presence of two additional novel transcripts in zebrafish,
$\operatorname{Ret}^{\Delta E 3}$ and Ret ${ }^{\Delta E 345}$, previously identified in various tumour sources and adult human tissues as a result of the skipping of exon 3 or exons 3, 4 and 5 has been reported (Gabreski et al. 2016). Commonly used reverse strategies to modulate the gene expression (morpholino-mediated knockdown, overexpression through mRNA injection and transgenesis) and recently developed ret mutants, harbouring activating mutations similar to those found in patients with MEN2, could provide relevant information on the molecular basis of MTC. Moreover, zebrafish could offer unique experimental conditions to clarify the potential role of $\mathrm{C}$ cells microenvironment in the MTC pathogenesis (Vitale et al. 2014). Although mammalian C cells are located in the thyroid interfollicular spaces, fish $\mathrm{C}$ cells form the ultimobranchial bodies, an independent organ that does not fuse with the thyroid. In zebrafish, starting from $60 \mathrm{~h}$ after fertilisation, ultimobranchial bodies are organised in two groups of cells on either side of the heart, in this way, C cells are not in contact with thyroid follicles (Porazzi et al. 2009). Thus, zebrafish could help us in understanding if extracellular matrix components, produced by thyroid epithelial, are necessary to the emergence of the MTC phenotype, as previously hypothesised in humans (Lekmine et al. 1999).

Another advantage of zebrafish model is offered by the possibility to use embryos, larvae and adults to perform tumour xenografts of immortalised cell lines or patient-derived cell culture, with the main aim of studying tumour-induced angiogenesis and invasiveness and performing antitumor drug screening. Recently, we have developed a system to study MTC-induced angiogenesis, based on the injection of human MTC cell lines in the $T g$ (fli1:EGFP)y1 zebrafish embryos that expresses EGFP (enhanced green fluorescent protein) under the control of the endothelial fli1 promoter, thereby labelling all blood vessels and providing a live visual marker for vascular development. In particular, we have grafted TT (Vitale et al. 2014) and MZ-CRC1 (Fig. 1) cell lines, which harbour MEN2A (C634W) and MEN2B (M918T) RET mutations, respectively. Red stained MTC cells (100-500 cells for embryo) were implanted into the subperidermal cavity (between the outer periderm and the yolk syncytial layer) of $48 \mathrm{~h}$ post-fertilisation $\mathrm{Tg}\left(\right.$ fli1:EGFP) ${ }^{y 1}$ zebrafish embryos. We evaluated the proangiogenic potential of grafted cells in a short time frame, from 24 to $48 \mathrm{~h}$ after injection, and without the need of immunosuppression, because the immune system is not completely developed during the first month of zebrafish life (Tobia et al. 2011). Although alterations of vascular network were absent in the control 

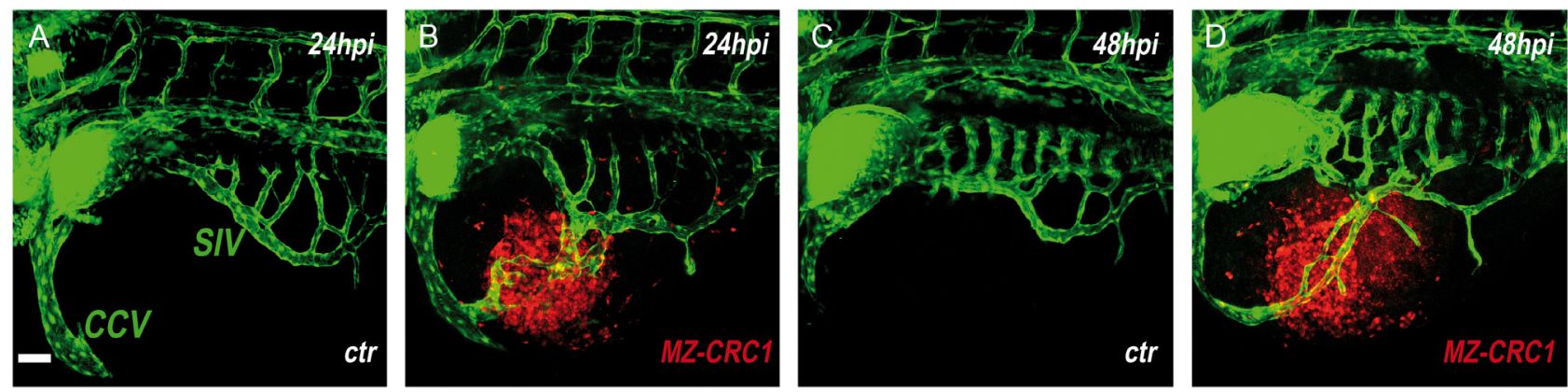

Figure 1

MTC-grafted cells stimulate angiogenesis in zebrafish embryos. Representative confocal microscopic images of $T g(f l i 1$ :EGFP)y1 zebrafish embryos implanted with red fluorescence-stained MZ-CRC1 ( $B$ and D) cells. 24 ( $A$ and B) and 48 ( $C$ and D) hours after injection (hpi), larvae were embedded in low-melting agarose, and the yolk region was observed by confocal microscopy. In comparison to control larvae (A and B), MTC-grafted larvae showed endothelial structures (green) that sprout from the subintestinal vein (SIV) plexus and the common cardinal vein (CCV) (B and D) and progressively form new vessels. All images are oriented so that rostral is to the left and dorsal is at the top. Scale bar, $50 \mu \mathrm{m}$.

group, consisting of phosphate buffered saline solution (PBS)-injected embryos, endothelial structures, sprouting from the subintestinal vein (SIV) and the common cardinal vein (CCV), were observed in TT- and MZ-CRC1grafted embryos (Fig. 1). These endothelial sprouts were progressively converted in vessels with heterogeneous diameter and length that could rapidly reach the implanted tumour cells mass. In these experimental conditions, TT cells showed a stronger proangiogenic potential than MZ-CRC-1. Indeed, a more intricate network of new blood vessels was observed in TT tumour-xenograft within 24-48 h after injection. In this model, the existence of MTC-specific angiogenic signalling pathways can also be analysed through a selective knockdown of receptors for proangiogenic factors in the recipient zebrafish embryos. In this way, it is possible to identify which proangiogenic factors, produced by MTC cells, have a predominant activity in tumour-induced angiogenesis.

Due to the easy and rapid visualisation of the tumour angiogenesis, zebrafish/tumour xenograft model represents a valuable platform to test the effects of antiangiogenic compounds on tumour-induced vascularisation. At the same time, invasiveness of MTC cells could be analysed within 2-3 days after the implant. Indeed, the transparency of the zebrafish embryos allows to follow the very early steps of invasion, circulation of tumour cells (stained with a fluorescent cell tracker) in blood vessels, colonisation at secondary organ sites and metastasis formation in real time. Therefore, this new animal model for MTC provides unique tools for the visualisation of tumour cell behaviour and interaction with host cells.

Zebrafish embryos are readily permeable to small molecules dissolved in their culture media. Therefore, the zebrafish/tumour xenograft model represents a rapid and suitable test to screen small molecules with potential antitumor activity and using a small amount of compounds, whereas larger or non-water-soluble molecules can be injected into the body of the embryo to ensure drug uptake. The rapidity of this procedure makes this model very useful to perform preclinical drug screening, to test and validate new compounds that are able to significantly inhibit tumour-induced angiogenesis, invasiveness and metastatic dissemination, as already described in several tumours implanted in zebrafish embryos (Moshal et al. 2011, Goel et al. 2013, Chen et al. 2015, Zheng et al. 2016).

Recently, our group sets up a method to generate PDX of neuroendocrine tumour cells in zebrafish embryos to study both the proangiogenic potential and the migratory behaviour of grafted cells (Gaudenzi et al. 2016, Wurth et al. 2016). The appeal of primary cultures, instead of immortalised cell line, lies in the conservation of both histological and mutational heterogeneity of the original tumour (Cassidy et al. 2015). The possibility to implant a small numbers of cells ( $<500$ cells for embryo) makes the application of PDX in zebrafish embryos particularly suitable for neuroendocrine tumours, where tumour cells availability is often limited, because of the small size of post-surgical samples. In this frame, PDX of MTC in zebrafish could represent a promising platform to develop new pharmacological treatments and to identify information for the most appropriate and personalised therapies.

However, there are also few disadvantages of using this model (Gaudenzi et al. 2016), that need to be considered, such as species-specific microenvironmental differences may affect the behaviour of grafted mammalian

Published by Bioscientifica Ltd 
tumour cells; the lack of some mammalian organs in fishes (such as mammary gland, prostate and lung) precludes the possibility to perform orthotopic transplantation experiments; drug metabolism in zebrafish may be different from that in mammals; zebrafish embryos are maintained at $28^{\circ} \mathrm{C}$, this may not represent an optimal temperature for mammalian cell growth and metabolism; embryonic organs and systems are completely defined but their differentiation is incomplete.

\section{Conclusions and future perspectives}

Animal models are indispensable tools for investigating the pathogenesis, pathophysiology, mechanisms for tumour invasion and metastasis and new therapeutic approaches for cancer. Unfortunately, only few models are available for MTC.

Murine models remain the gold standard for preclinical study in MTC. RET transgenic mouse models play a relevant role in understanding the pathogenesis and progression of this tumour. MTC xenograft mouse models are crucial to perform preclinical drug screening, but are limited to the implantation of few MTC cell lines. PDX represents a more realistic preclinical model. Indeed, PDX preserves the original tumour identity, reflecting more accurately the complexity of human tumour composition, architecture and development. However, there are several limitations associated with PDX murine model in MTC and in other tumours with low proliferation index: low engraftment rates, long time required (from several weeks to months) to have a visible tumour implant, large number of cells (about 1 million) are required to generate a tumour, and high difficulties to generate xenotransplant models able to metastasise in mouse. In addition, immunosuppressed mice are required to avoid transplant rejection, these animals are more susceptible to infection and drug toxicity than normal mice and need special standards for breeding and housing; its laborious and time-consuming process makes this model very expensive.

Other animal organisms have been recently proposed as alternative or complementary models to mice. Zebrafish/tumour xenograft model represents an attractive, fast and technically simple model to study tumour-host microenvironment, to better characterise the multiple mechanisms of angiogenesis and invasiveness in MTC and to test in vivo the antitumor effects of new compounds. The short generation time, the large number of offspring, the transparency (enabling noninvasive imaging), the external development of the embryos and the small size make zebrafish a more practical and less expensive laboratory system than other in vivo cancer models.

Preliminary data suggest the PDX in zebrafish embryos appears to be a reliable in vivo preclinical model for neuroendocrine tumours (Gaudenzi et al. 2016, Wurth et al. 2016), opening a future scenario for personalised treatment in patients with MTC.

Over the last years, several innovations in live imaging of zebrafish embryos have been developed to study embryogenesis. In addition to the conventional applications in epifluorescence, confocal and multiphoton microscopy, zebrafish has recently resulted a valid platform for selective plane illumination microscopy (SPIM) (Kaufmann et al. 2012), a technique that achieves excellent resolution at high penetration depth in translucent samples. The most prominent advantages of SPIM is the high acquisition speed and the reduced photo-toxicity, making this technique particularly suitable for long-term timelapse experiments in live samples (Huisken \& Stainier 2009). SPIM has been successfully used on zebrafish embryos for high-resolution time-lapse imaging of developmental processes lasting several hours to a few days (Panier et al. 2013, Mickoleit et al. 2014, Bassi et al. 2015), as shown in Supplementary Video 1, see section on supplementary data given at the end of this article. In future, this innovative technique could provide new information for the study of the tumour-host interactions in MTC, with particular interest towards tumour-induced angiogenesis and tumour invasiveness. Indeed, SPIM would offer the possibility to localise, track and register MTC cells and their interactions with the local microenvironment.

Finally, all these tumour xenograft models lack the innate and adaptive immune system that both play an important role in tumour development and progression. In this regard, the development of hemato-lymphoid humanised models, such as through the cotransplantation of MTC cells as well as human immune or stromal cells into an immunodeficient animal, may represent a future promising strategy for the study of this tumour, especially concerning immunological processes and for testing new immunotherapies against tumour.

\footnotetext{
Supplementary data
}

This is linked to the online version of the paper at http://dx.doi.org/10.1530/ ERC-16-0399. 


\section{Declaration of interest}

The authors declare that there is no conflict of interest that could be perceived as prejudicing the impartiality of this review.

\section{Funding}

This work was partially supported by the Italian Ministry of Education, Research and University (FIRB RBAP11884M).

\section{Acknowledgements}

This review is part of the 'NIKE' project (Neuroendocrine tumors Innovation Knowledge and Education) led by Prof Annamaria Colao, which aims at increasing the knowledge on NETs. The authors would like to acknowledge all the Collaborators of this project: Albertelli M, Amoroso $\mathrm{V}$, Arvat E, Badalamenti G, Bajetta E, Baldelli R, Bianchi A, Botti G, Buzzoni R, Campana D, Carnaghi C, Cartenì C, Cingarlini S, Colantuoni V, Coppa J, Corcione F, Davi M V, De Rosa G, degli Uberti E, Del Basso De Caro M L, Di Sarno A, Falconi M, Fanciulli G, Ferolla P, Ferone D, Fiore F, Gallo M, Giordano C, Giuffrida D, Grossrubatscher E M, Guadagno E, Guarnotta V, Izzo F, Lania A, Lastoria S, Leo S, Lo Calzo F, Logoluso F, Malandrino P, Marchetti M, Martini C, Messina E, Milione M, Modica R, Montesarchio V, Muto $P$, Nappi O, Nazzari E, Panzuto F, Pia A, Piovesan A, Pizza G, Pontecorvi A, Pusceddu S, Razzore P, Riccardi F, Rindi G, Rota F, Sciammarella C, Spada F, Tafuto S, Versari A, Volante M and Zatelli M C.

\section{References}

Acton DS, Velthuyzen D, Lips CJ \& Hoppener JW 2000 Multiple endocrine neoplasia type $2 \mathrm{~B}$ mutation in human RET oncogene induces medullary thyroid carcinoma in transgenic mice. Oncogene 19 3121-3125. (doi:10.1038/sj.onc.1203648)

Akeno N, Miller AL, Ma X \& Wikenheiser-Brokamp KA 2015 p53 suppresses carcinoma progression by inhibiting mTOR pathway activation. Oncogene 34 589-599. (doi:10.1038/onc.2013.589)

Anwar F, Emond MJ, Schmidt RA, Hwang HC \& Bronner MP 2000. Retinoblastoma expression in thyroid neoplasms. Modern Pathology 13 562-569. (doi:10.1038/modpathol.3880097)

Bassi A, Schmid B \& Huisken J 2015 Optical tomography complements light sheet microscopy for in toto imaging of zebrafish development. Development 142 1016-1020. (doi:10.1242/dev.116970)

Bentzien F, Zuzow M, Heald N, Gibson A, Shi Y, Goon L, Yu P, Engst S, Zhang W, Huang D, et al. 2013 In vitro and in vivo activity of cabozantinib (XL184), an inhibitor of RET, MET, and VEGFR2, in a model of medullary thyroid cancer. Thyroid 23 1569-1577. (doi:10.1089/thy.2013.0137)

Broutin S, Ameur N, Lacroix L, Robert T, Petit B, Oumata N, Talbot M, Caillou B, Schlumberger M, Dupuy C, et al. 2011 Identification of soluble candidate biomarkers of therapeutic response to sunitinib in medullary thyroid carcinoma in preclinical models. Clinical Cancer Research 17 2044-2054. (doi:10.1158/1078-0432.CCR-10-2041)

Cabanillas ME, McFadden DG \& Durante C 2016 Thyroid cancer. Lancet [in press]. (doi:10.1016/s0140-6736(16)30172-6)

Cassidy JW, Caldas C \& Bruna A 2015 Maintaining tumor heterogeneity in patient-derived tumor xenografts. Cancer Research 75 2963-2968. (doi:10.1158/0008-5472.CAN-15-0727)

Cerrato A, De Falco V \& Santoro M 2009 Molecular genetics of medullary thyroid carcinoma: the quest for novel therapeutic targets. Journal of Molecular Endocrinology 43 143-155. (doi:10.1677/JME-09-0024)

Chen X, Wang J, Cao Z, Hosaka K, Jensen L, Yang H, Sun Y, Zhuang R, Liu Y \& Cao Y 2015 Invasiveness and metastasis of retinoblastoma in an orthotopic zebrafish tumor model. Scientific Reports 510351. (doi:10.1038/srep10351)

Cote GJ, Grubbs EG \& Hofmann MC 2015 Thyroid C-cell biology and oncogenic transformation. Recent Results in Cancer Research 204 1-39. (doi:10.1007/978-3-319-22542-5_1)

Coxon AB, Ward JM, Geradts J, Otterson GA, Zajac-Kaye M \& Kaye FJ 1998 RET cooperates with RB/p53 inactivation in a somatic multistep model for murine thyroid cancer. Oncogene 17 1625-1628. (doi:10.1038/sj.onc.1202381)

Cranston AN \& Ponder BA 2003 Modulation of medullary thyroid carcinoma penetrance suggests the presence of modifier genes in a RET transgenic mouse model. Cancer Research 63 4777-4780.

Dar AC, Das TK, Shokat KM \& Cagan RL 2012 Chemical genetic discovery of targets and anti-targets for cancer polypharmacology. Nature 486 80-84. (doi:10.1038/nature11127)

Das T \& Cagan R 2010 Drosophila as a novel therapeutic discovery tool for thyroid cancer. Thyroid 20 689-695. (doi:10.1089/thy.2010.1637)

Das SS, Nanda GG \& Alone DP 2014 Artemisinin and curcumin inhibit Drosophila brain tumor, prolong life span, and restore locomotor activity. IUBMB Life 66 496-506. (doi:10.1002/iub.1284)

Druce M, Chung TT, Grozinsky-Glasberg S, Gross DJ \& Grossman AB 2012 Preliminary report of the use of everolimus in a patient with progressive medullary thyroid carcinoma. Clinical Endocrinology $\mathbf{7 7}$ 154-155. (doi:10.1111/j.1365-2265.2011.04296.x)

Faggiano A, Ramundo V, Dicitore A, Castiglioni S, Borghi MO, Severino R, Ferolla P, Crino L, Abbruzzese A, Sperlongano P, et al. 2012 Everolimus is an active agent in medullary thyroid cancer: a clinical and in vitro study. Journal of Cellular and Molecular Medicine 16 1563-1572. (doi:10.1111/j.1582-4934.2011.01438.x)

Fisher S, Grice EA, Vinton RM, Bessling SL \& McCallion AS 2006 Conservation of RET regulatory function from human to zebrafish without sequence similarity. Science 312 276-279. (doi:10.1126/ science.1124070)

Franklin DS, Godfrey VL, O’Brien DA, Deng C \& Xiong Y 2000 Functional collaboration between different cyclin-dependent kinase inhibitors suppresses tumor growth with distinct tissue specificity. Molecular and Cellular Biology 20 6147-6158. (doi:10.1128/ MCB.20.16.6147-6158.2000)

Gabreski NA, Vaghasia JK, Novakova SS, McDonald NQ \& Pierchala BA 2016 Exon skipping in the RET gene encodes novel isoforms that differentially regulate RET protein signal transduction. Journal of Biological Chemistry 291 16249-16262. (doi:10.1074/jbc. M115.709675)

Gaudenzi G, Albertelli M, Dicitore A, Wurth R, Gatto F, Barbieri F, Cotelli F, Florio T, Ferone D, Persani L, et al. 2016 Patient-derived xenograft in zebrafish embryos: a new platform for translational research in neuroendocrine tumors. Endocrine [in press]. (doi:10.1007/s12020-016-1048-9)

Goel S, Gupta N, Walcott BP, Snuderl M, Kesler CT, Kirkpatrick ND, Heishi T, Huang Y, Martin JD, Ager E, et al. 2013 Effects of vascularendothelial protein tyrosine phosphatase inhibition on breast cancer vasculature and metastatic progression. Journal of the National Cancer Institute 105 1188-1201. (doi:10.1093/jnci/djt164)

Grubbs EG, Williams MD, Scheet P, Vattathil S, Perrier N, Lee JE, Gagel RF, Hai T, Feng L, Cabanillas M, et al. 2016 The role of CDKN2C copy number in sporadic medullary thyroid carcinoma. Thyroid 26 1553-1562. (doi:10.1089/thy.2016.0224)

Gyory F, Mezosi E, Szakall S, Bajnok L, Varga E, Borbely A, Gazdag A, Juhasz I, Lukacs G \& Nagy EV 2005 Establishment of the hu-PBLSCID mouse model for the investigation of thyroid cancer. Experimental and Clinical Endocrinology and Diabetes 113 359-364. (doi:10.1055/s-2005-865740)

Harrison DJ, Hooper ML, Armstrong JF \& Clarke AR 1995 Effects of heterozygosity for the Rb-1t19neo allele in the mouse. Oncogene $\mathbf{1 0}$ $1615-1620$
(C) 2017 Society for Endocrinology Printed in Great Britain
Published by Bioscientifica Ltd 
Harvey M, Vogel H, Lee EY, Bradley A \& Donehower LA 1995 Mice deficient in both p53 and Rb develop tumors primarily of endocrine origin. Cancer Research 55 1146-1151.

Holm R \& Nesland JM 1994 Retinoblastoma and p53 tumour suppressor gene protein expression in carcinomas of the thyroid gland. Journal of Pathology 172 267-272. (doi:10.1002/path.1711720307)

Huisken J \& Stainier DY 2009 Selective plane illumination microscopy techniques in developmental biology. Development 136 1963-1975. (doi:10.1242/dev.022426)

Huiting LN, Laroche F \& Feng H 2015 The zebrafish as a tool to cancer drug discovery. Austin Journal of Pharmacology and Therapeutics $\mathbf{3}$ 1069.

Johnston D, Hatzis D \& Sunday ME 1998 Expression of v-Ha-ras driven by the calcitonin/calcitonin gene-related peptide promoter: a novel transgenic murine model for medullary thyroid carcinoma. Oncogene 16 167-177. (doi:10.1038/sj.onc.1201478)

Kaufmann A, Mickoleit M, Weber M \& Huisken J 2012 Multilayer mounting enables long-term imaging of zebrafish development in a light sheet microscope. Development 139 3242-3247. (doi:10.1242/ dev.082586)

Kebebew E, Greenspan FS, Clark OH, Woeber KA \& Grunwell J 2005 Extent of disease and practice patterns for medullary thyroid cancer. Journal of the American College of Surgeons 200 890-896. (doi:10.1016/j.jamcollsurg.2004.12.011)

Kim MP, Evans DB, Wang H, Abbruzzese JL, Fleming JB \& Gallick GE 2009 Generation of orthotopic and heterotopic human pancreatic cancer xenografts in immunodeficient mice. Nature Protocols 4 1670-1680. (doi:10.1038/nprot.2009.171)

Kirschner LS, Qamri Z, Kari S \& Ashtekar A 2016 Mouse models of thyroid cancer: a 2015 update. Molecular and Cellular Endocrinology 421 18-27. (doi:10.1016/j.mce.2015.06.029)

Lekmine F, Feracci H, Milhaud G, Treilhou-Lahille F \& Jeanne N 1999 Expression of laminin-2 by normal and neoplastic rat $\mathrm{C}$ cells during the development of medullary thyroid carcinoma. Virchows Archiv 434 325-332. (doi:10.1007/s004280050348)

Levinson S \& Cagan RL 2016 Drosophila cancer models identify functional differences between Ret fusions. Cell Reports $\mathbf{1 6}$ 3052-3061. (doi:10.1016/j.celrep.2016.08.019)

Lim SM, Chang H, Yoon MJ, Hong YK, Kim H, Chung WY, Park CS, Nam KH, Kang SW, Kim MK, et al. 2013 A multicenter, phase II trial of everolimus in locally advanced or metastatic thyroid cancer of all histologic subtypes. Annals of Oncology 24 3089-3094. (doi:10.1093/ annonc/mdt379)

Lopergolo A, Nicolini V, Favini E, Dal Bo L, Tortoreto M, Cominetti D, Folini M, Perego P, Castiglioni V, Scanziani E, et al. 2014 Synergistic cooperation between sunitinib and cisplatin promotes apoptotic cell death in human medullary thyroid cancer. Journal of Clinical Endocrinology and Metabolism 99 498-509. (doi:10.1210/ jc.2013-2574)

Lucini C, D'Angelo L, Patruno M, Mascarello F, de Girolamo P \& Castaldo L 2011 GDNF family ligand RET receptor in the brain of adult zebrafish. Neuroscience Letters 502 214-218. (doi:10.1016/j. neulet.2011.07.047)

Lupoli G, Cascone E, Arlotta F, Vitale G, Celentano L, Salvatore M \& Lombardi G 1996 Treatment of advanced medullary thyroid carcinoma with a combination of recombinant interferon alpha-2b and octreotide. Cancer 78 1114-1118. (doi:10.1002/(SICI)10970142(19960901)78:5<1114::AID-CNCR23>3.0.CO;2-4)

Manfredi GI, Dicitore A, Gaudenzi G, Caraglia M, Persani L \& Vitale G 2016 Erratum to: PI3K/Akt/mTOR signaling in medullary thyroid cancer: a promising molecular target for cancer therapy. Endocrine $\mathbf{5 3}$ 874. (doi:10.1007/s12020-016-1000-z)

Marcos-Gutierrez CV, Wilson SW, Holder N \& Pachnis V 1997 The zebrafish homologue of the ret receptor and its pattern of expression during embryogenesis. Oncogene 14 879-889. (doi:10.1038/sj. onc.1201048)
Michiels FM, Chappuis S, Caillou B, Pasini A, Talbot M, Monier R, Lenoir GM, Feunteun J \& Billaud M 1997 Development of medullary thyroid carcinoma in transgenic mice expressing the RET protooncogene altered by a multiple endocrine neoplasia type $2 \mathrm{~A}$ mutation. PNAS 94 3330-3335. (doi:10.1073/pnas.94.7.3330)

Mickoleit M, Schmid B, Weber M, Fahrbach FO, Hombach S, Reischauer S \& Huisken J 2014 High-resolution reconstruction of the beating zebrafish heart. Nature Methods 11 919-922. (doi:10.1038/ nmeth.3037)

Modigliani E, Cohen R, Campos JM, Conte-Devolx B, Maes B, Boneu A, Schlumberger M, Bigorgne JC, Dumontier P, Leclerc L, et al. 1998 Prognostic factors for survival and for biochemical cure in medullary thyroid carcinoma: results in 899 patients. The GETC Study Group. Groupe d'etude des tumeurs a calcitonine. Clinical Endocrinology $\mathbf{4 8}$ 265-273. (doi:10.1046/j.1365-2265.1998.00392.x)

Moshal KS, Ferri-Lagneau KF, Haider J, Pardhanani P \& Leung T 2011 Discriminating different cancer cells using a zebrafish in vivo assay. Cancers 3 4102-4113. (doi:10.3390/cancers3044102)

Moura MM, Cavaco BM \& Leite V 2015 RAS proto-oncogene in medullary thyroid carcinoma. Endocrine-Related Cancer 22 R235-R252. (doi:10.1530/ERC-15-0070)

Myers SM, Eng C, Ponder BA \& Mulligan LM 1995 Characterization of RET proto-oncogene 3 ' splicing variants and polyadenylation sites: a novel C-terminus for RET. Oncogene 11 2039-2045.

Panier T, Romano SA, Olive R, Pietri T, Sumbre G, Candelier R \& Debregeas G 2013 Fast functional imaging of multiple brain regions in intact zebrafish larvae using selective plane illumination microscopy. Frontiers in Neural Circuits 7 65. (doi:10.3389/ fncir.2013.00065)

Park MS, Rosai J, Nguyen HT, Capodieci P, Cordon-Cardo C \& Koff A 1999 p27 and Rb are on overlapping pathways suppressing tumorigenesis in mice. PNAS 96 6382-6387. (doi:10.1073/ pnas.96.11.6382)

Pavelić K, Dedivitis RA, Kapitanović S, Cacev T, Guirado CR, Danić D, Radosević S, Brkić K, Pegan B, Krizanac S, et al. 2006 Molecular genetic alterations of FHIT and p53 genes in benign and malignant thyroid gland lesions. Mutation Research 599 45-57. (doi:10.1016/j. mrfmmm.2006.01.021)

Porazzi P, Calebiro D, Benato F, Tiso N \& Persani L 2009 Thyroid gland development and function in the zebrafish model. Molecular and Cellular Endocrinology 312 14-23. (doi:10.1016/j. mce.2009.05.011)

Pozo K, Castro-Rivera E, Tan C, Plattner F, Schwach G, Siegl V, Meyer D, Guo A, Gundara J, Mettlach G, et al. 2013 The role of Cdk5 in neuroendocrine thyroid cancer. Cancer Cells 24 499-511. (doi:10.1016/j.ccr.2013.08.027)

Read RD, Goodfellow PJ, Mardis ER, Novak N, Armstrong JR \& Cagan RL 2005 A Drosophila model of multiple endocrine neoplasia type 2 . Genetics 171 1057-1081. (doi:10.1534/genetics.104.038018)

Reynolds L, Jones K, Winton DJ, Cranston A, Houghton C, Howard L, Ponder BA \& Smith DP 2001 C-cell and thyroid epithelial tumours and altered follicular development in transgenic mice expressing the long isoform of MEN 2A RET. Oncogene 20 3986-3994. (doi:10.1038/ sj.onc.1204434)

Ribatti D 2014 The chick embryo chorioallantoic membrane as a model for tumor biology. Experimental Cell Research 328 314-324. (doi:10.1016/j.yexcr.2014.06.010)

Ribatti D 2016 The chick embryo chorioallantoic membrane (CAM). A multifaceted experimental model. Mechanisms of Development 141 70-77. (doi:10.1016/j.mod.2016.05.003)

Roman S, Lin R \& Sosa JA 2006 Prognosis of medullary thyroid carcinoma: demographic, clinical, and pathologic predictors of survival in 1252 cases. Cancer 107 2134-2142. (doi:10.1002/cncr.22244)

Romei C, Ciampi R \& Elisei R 2016 A comprehensive overview of the role of the RET proto-oncogene in thyroid carcinoma. Nature Reviews Endocrinology 12 192-202. (doi:10.1038/nrendo.2016.11) 
Rubio-Viqueira B \& Hidalgo M 2009 Direct in vivo xenograft tumor model for predicting chemotherapeutic drug response in cancer patients. Clinical Pharmacology and Therapeutics 85 217-221. (doi:10.1038/clpt.2008.200)

Santoro M \& Carlomagno F 2013 Central role of RET in thyroid cancer. Cold Spring Harbor Perspectives in Biology 5 a009233. (doi:10.1101/ cshperspect.a009233)

Schneider TC, de Wit D, Links TP, van Erp NP, van der Hoeven JJ, Gelderblom H, van Wezel T, van Eijk R, Morreau H, Guchelaar HJ, et al. 2015 Beneficial effects of the mTOR inhibitor everolimus in patients with advanced medullary thyroid carcinoma: subgroup results of a phase II trial. International Journal of Endocrinology 2015 348124. (doi:10.1155/2015/348124)

Sheikh HA, Tometsko M, Niehouse L, Aldeeb D, Swalsky P, Finkelstein S, Barnes EL \& Hunt JL 2004 Molecular genotyping ofmedullary thyroid carcinoma can predict tumor recurrence. American Journal of Surgical Pathology 28 101-106. (doi:10.1097/00000478-20040100000012)

Smith-Hicks CL, Sizer KC, Powers JF, Tischler AS \& Costantini F 2000 C-cell hyperplasia, pheochromocytoma and sympathoadrenal malformation in a mouse model of multiple endocrine neoplasia type 2B. EMBO Journal 19 612-622. (doi:10.1093/emboj/19.4.612)

Staton CA, Reed MW \& Brown NJ 2009 A critical analysis of current in vitro and in vivo angiogenesis assays. International Journal of Experimental Pathology 90 195-221. (doi:10.1111/ j.1365-2613.2008.00633.x)

Tobia C, De Sena G \& Presta M 2011 Zebrafish embryo, a tool to study tumor angiogenesis. International Journal of Developmental Biology $\mathbf{5 5}$ 505-509. (doi:10.1387/ijdb.103238ct)

Tohyama O, Matsui J, Kodama K, Hata-Sugi N, Kimura T, Okamoto K, Minoshima Y, Iwata M \& Funahashi Y 2014 Antitumor activity of lenvatinib (e7080): an angiogenesis inhibitor that targets multiple receptor tyrosine kinases in preclinical human thyroid cancer models. Journal of Thyroid Research 2014638747. (doi:10.1155/2014/638747)

van Oevelen C, Wang J, Asp P, Yan Q, Kaelin WG Jr., Kluger Y \& Dynlacht BD 2008 A role for mammalian Sin3 in permanent gene silencing. Molecular Cell 32 359-370. (doi:10.1016/j.molcel.2008.10.015)

van Veelen W, van Gasteren CJ, Acton DS, Franklin DS, Berger R, Lips CJ \& Hoppener JW 2008 Synergistic effect of oncogenic RET and loss of p18 on medullary thyroid carcinoma development. Cancer Research 68 1329-1337. (doi:10.1158/0008-5472.CAN-07-5754)

Vidal M, Wells S, Ryan A \& Cagan R 2005 ZD6474 suppresses oncogenic RET isoforms in a Drosophila model for type 2 multiple endocrine neoplasia syndromes and papillary thyroid carcinoma. Cancer Research 65 3538-3541. (doi:10.1158/0008-5472.CAN-04-4561)

Viola D, Valerio L, Molinaro E, Agate L, Bottici V, Biagini A, Lorusso L, Cappagli V, Pieruzzi L, Giani C, et al. 2016 Treatment of advanced thyroid cancer with targeted therapies: ten years of experience. Endocrine-Related Cancer 23 R185-R205. (doi:10.1530/ERC-15-0555)

Vitale G, Tagliaferri P, Caraglia M, Rampone E, Ciccarelli A, Bianco AR, Abbruzzese A \& Lupoli G 2000 Slow release lanreotide in combination with interferon-alpha2b in the treatment of symptomatic advanced medullary thyroid carcinoma. Journal of Clinical Endocrinology and Metabolism 85 983-988. (doi:10.1210/ jcem.85.3.6435)

Vitale G, Caraglia M, Ciccarelli A, Lupoli G, Abbruzzese A \& Tagliaferri P 2001 Current approaches and perspectives in the therapy of medullary thyroid carcinoma. Cancer 91 1797-1808. (doi:10.1002/10970142(20010501)91:9<1797::AID-CNCR1199>3.0.CO;2-P)

Vitale G, Gaudenzi G, Dicitore A, Cotelli F, Ferone D \& Persani L 2014 Zebrafish as an innovative model for neuroendocrine tumors. Endocrine-Related Cancer 21 R67-R83. (doi:10.1530/ERC-13-0388)

White R, Rose K \& Zon L 2013 Zebrafish cancer: the state of the art and the path forward. Nature Reviews Cancer 13 624-636. (doi:10.1038/ nrc3589)

Wiedemann T \& Pellegata NS 2016 Animal models of multiple endocrine neoplasia. Molecular and Cellular Endocrinology 421 49-59. (doi:10.1016/j.mce.2015.07.004)

Williams BO, Remington L, Albert DM, Mukai S, Bronson RT \& Jacks T 1994 Cooperative tumorigenic effects of germline mutations in $\mathrm{Rb}$ and p53. Nature Genetics 7 480-484. (doi:10.1038/ng0894-480)

Wurth R, Barbieri F, Pattarozzi A, Gaudenzi G, Gatto F, Fiaschi P, Ravetti JL, Zona G, Daga A, Persani L, et al. 2016 Phenotypical and pharmacological characterization of stem-like cells in human pituitary adenomas. Molecular Neurobiology [in press]. (doi:10.1007/ s12035-016-0025-x)

Yadav AK, Srikrishna S \& Gupta SC 2016 Cancer drug development using drosophila as an in vivo tool: from bedside to bench and back. Trends in Pharmacological Sciences 37 789-806. (doi:10.1016/j. tips.2016.05.010)

Yalcin M, Dyskin E, Lansing L, Bharali DJ, Mousa SS, Bridoux A, Hercbergs AH, Lin HY, Davis FB, Glinsky GV, et al. 2010 Tetraiodothyroacetic acid (tetrac) and nanoparticulate tetrac arrest growth of medullary carcinoma of the thyroid. Journal of Clinical Endocrinology and Metabolism 95 1972-1980. (doi:10.1210/jc.2009-1926)

Zheng MW, Zhang CH, Chen K, Huang M, Li YP, Lin WT, Zhang RJ, Zhong L, Xiang R, Li LL, et al. 2016 Preclinical evaluation of a novel orally available SRC/Raf/VEGFR2 inhibitor, SKLB646, in the treatment of triple-negative breast cancer. Molecular Cancer Therapeutics 15 366-378. (doi:10.1158/1535-7163.MCT-15-0501)

Ziebold U, Lee EY, Bronson RT \& Lees JA 2003 E2F3 loss has opposing effects on different pRB-deficient tumors, resulting in suppression of pituitary tumors but metastasis of medullary thyroid carcinomas. Molecular and Cellular Biology 23 6542-6552. (doi:10.1128/ МСB.23.18.6542-6552.2003)

Received in final form 18 October 2016

Accepted 24 October 2016

Accepted Preprint published online 31 October 2016
๑ 2017 Society for Endocrinology Printed in Great Britain
Published by Bioscientifica Ltd. 